\title{
The Construction of the Mobile E-commerce Precision Marketing Model Based on SoLoMo
}

\author{
Bin $\mathrm{Gu}^{1, a}$, Weisha Cai ${ }^{2, \mathrm{~b},{ }^{*}}$
}

${ }^{1}$ School of Economics and Commerce, South China University of Technology, 510006, Guangdong, China

${ }^{2}$ School of Economics and Commerce, South China University of Technology, 510006, Guangdong, China

a69985608@qq.com, b892111299@qq.com

Keywords: SoLoMo, Mobile E-commerce, Precision Marketing.

\begin{abstract}
With the advent of the era of mobile Internet, the opportunities of mobile e-commerce and mobile marketing are growing. After the analysis of the reason to construct the precision marketing model based on SoLoMo, the paper combines social networks, location-based services with mobile technology, builds the systemic mobile e-commerce precision marketing model based on SoLoMo from the three aspects, which is advantageous to the merchants to carry out precision marketing and consumers to get needed information in a timely manner.
\end{abstract}

\section{基于SoLoMo的移动电子商务精准化营销模型构建 \\ 谷斌 $1, a$, 蔡玮莎 $2, b,{ }^{*}$ \\ 1华南理工大学经济与贸易学院, 广州市, 广东省, 中国 \\ 2华南理工大学经济与贸易学院, 广州市, 广东省, 中国 \\ a69985608@qq.com, b892111299@qq.com}

关键词: SoLoMo; 移动电子商务; 精准营销

中文摘要. 随着移动互联网时代的到来, 移动电子商务和移动营销的机会在不断增长。本文 在分析构建基于SoLoMo的精准化营销模型的原因后, 将社交网络、基于位置的服务和移动技 术结合, 从这三个方面构建系统的基于SoLoMo的移动电子商务精准化营销模型, 有利于商家 开展精准化营销，消费者及时获知需要的信息。

\section{1. 引言}

随着移动互联网的迅猛崛起，人们的社交范围通过网络变得更广泛，移动智能终端提供 了随时随地的沟通，用户之间、商家与消费者之间，信息搜集、发送、传播、分享也变得更 顺畅。越来越多的人接入移动互联网, 从近年来 “双十一” 移动端购物的数据不断攀升就可 以看出移动互联网市场的发展潜力。另外, 4G信号的覆盖, 流量费用的下调, 5G网络的开拓, 以及公共场所越来越多的Wi-Fi热点的建设，人们消费习惯的改变等，这些都十分有利于人们 进行网络消费。传统的电信运营商例如中国移动也更加重视移动业务的开发, 大众创业的热 情也推动了更多开展手机团购等企业的发展, 未来, 移动电子商务的发展空间还很大, 能进 一步挖掘出消费者的消费潜力, 满足其个性化消费需求。伴随着人际交互的扩大, 已有的基 于LBS (Location Based Service)的移动营销理论和实践已经不够。基于SoLoMo的移动电子商 
务精准化营销才更符合发展趋势, 更有利于满足人与环境的交互需求, 更有利于推动我国移 动电子商务的发展, 更有利于全球经济的腾飞。

\section{2. 研究综述}

目前，移动互联网和移动电子商务的发展对传统的各行各业产生了巨大的影响，关于移 动电子商务精准化营销的研究越来越得到专家学者的极大关注。已有的研究主要可以分为两 大类。

第一类是对基于LBS的移动电子商务的营销模型和策略的研究。这里要提到的是，移动 电子商务是指依托无线互联网、移动客户端等的企业与消费者之间的电子商务活动。精准营 销则是在精准定位的基础上更注重结果和行动的营销传播计划。卢秋萍在分析了移动电子商 务精准化营销优势的基础上，提出了基于LBS的移动电子商务个性化定价策略和移动促销模 型, 并指出在实施策略方面, 应该加大政府扶持力度、加强移动电子商务消费者的市场认知 教育等 ${ }^{[1]}$ 。黄辰伟以中国小商品城为例, 研究了描述需求、系统功能、应用场景、商务模式、 短信应用的基础上的移动顾客导购系统 ${ }^{[2]}$ 。这类研究能够从移动营销的精要出发, 但都是基 于LBS的, 没有结合社交网络和移动终端、移动网络进行分析, 研究范围和深度存在深化扩 大的空间。

第二类研究是基于 SoLoMo 的概念营销。这方面国外学者的研究比较领先。关于 SoLoMo(Social+Local+Mobile)的理念, 最初可追溯到美国最大的风险基金凯鹏华盈(KPCB)的 合伙人约翰・杜尔(John Doerr)的《移动互联网的十大发展趋势》(Top 10 Mobile Internet Trends) 的演讲。其中, Social能够聚焦人气; Local是地理基础, 提供移动终端应用中的基于地理位 置的信息服务; Mobile则是技术支撑、基础和载体方面的概括 ${ }^{[3]}$ 。后来，随着4G、TD-LTE等 移动互联网技术的发展, 移动社交媒体、移动RSS、移动交流软件为用户提供了更便利的信 息获取方式和分享环境。基于此，电通公司提出了一种新的消费者行为分析模型一一ASAS 模型, 认为现在品牌的营销模式开始向AISAS模式转变, 这种模式具有一定的网络特质 ${ }^{[4]}$ 。 它更强调以用户需求为中心, 其突出的关键词为 “精准营销(Search)” 和 “即时共享(Share)”。 这类研究综合运用了SoLoMo的概念, 但还是不够完善; 强调了概念营销和其混搭运用, 而忽 略了更为系统的研究和精准化营销模型的构建。

\section{3. 构建基于SoLoMo的精准化营销模型的原因}

\subsection{SoLoMo时代企业面临新挑战}

SoLoMo极大地改变了信息的搜集、传播、共享方式，从根本上改善了人们互动交流的局 限性, 也改变了企业与消费者的对接模式。SoLoMo的发展对于企业营销而言, 符合企业的产 品设计、计划、品牌传播和目标受众。也就是说, 企业的品牌营销也从单向的传达变为多向 的融入消费者的日常生活互动。品牌在传播的过程中, 也可以达到加强消费者联系的作用, 通过社会化媒体的一些应用和社交网络, 使消费者不仅是进行物质或精神消费, 也产生了情 感认同，对维护客户的忠诚度和促进再次购买有重大意义。消费者越来越渴望与周围环境、 系统产生交互作用或互动, 找到满足自己个性化需求的消费或者信息。这种双向互动关系, 不仅体现在消费者或个体对外界信息的获取和利用，也体现在行动之后的 “分享”过程。

同时，大部分企业的营销人员对SoLoMo营销的概念还很不明晰，或者说很多企业都还没 有开展SoLoMo营销的大方向把握，还没有意识到SoLoMo精准化营销的重要性或者说意识到 了却不知如何入手开展, 以及缺乏如何进行有效的关联而建设成为立体的SoLoMo营销网络的 经验。 


\subsection{SoLoMo精准化营销的优势}

首先, 构建基于SoLoMo的移动电子商务精准化营销模型, 一方面, 有利于借助So的方面 全面增强卖家与顾客的社会和情感联系。从商家的角度而言, 有利于开展一对一的精准式营 销。通过及时推送个性化的比较精确的广告信息, 以及对消费者的购物或消费记录信息的收 集, 能够调动消费者的消费需求转换为消费需要, 再转化为购买欲望, 从而提升顾客服务。 从消费者的角度分析, 有利于消费者随时随地获知营销信息, 更加感受到自己享受的服务是 独特优质的, 进行消费得到客户效用与价值。另一方面, 基于SoLoMo的移动电子商务精准化 营销更容易增加消费者的忠诚度。借助于So也即社交网络，更多的消费者借鉴他人的评价进 行消费或参与其他体验, 也有更多的人主动地将自己的消费感受或体验评价发布在社交媒体 上, 这种口碑相传的作用不容小觑。据调查, 大部分消费者在网购时, 更趋向于使用移动端, 因为有更多的优惠或折扣，在消费新鲜的服务时也很愿意参考其他消费者的评价或感言。

其次, Lo也即基于LBS的服务使得定位更加精准。例如, 用户可通过GPS对附近有什么商 家或者便利服务的地点、促销信息进行搜索。基于位置的服务将是未来移动电子商务的发展 重点, 能够进行一站式的服务。对于商家来说, 基于位置的推送服务更加精准, 还可以结合 周边社区进行促销，甚至可以结合微信新推出的支付现金反馈激励（可以在附近门店使用）、 支付宝新推出的AR实景红包等进行营销; 也可以对一些门店经常关顾的客户提供签到或积分 等激励, 进一步活跃客户。另外, 基于位置的服务可以与 $\mathrm{O} 2 \mathrm{O}$ 模式相结合, 即实现线上线下 的互动, 提供一种综合服务性模式, 集亲子休闲、观影唱歌等娱乐、虚拟现实购物等为一体 的生活体验模式。线下配备相关的上网和娱乐设施, 提供便利生活服务和消费体验, 实现线 上客户为线下引流, 线下客户又向线上转化, 扩大用户群的效果。例如具有品牌、服务和网 络优势的一些大型服装门店就可以采取这种营销模式[5]。

最后, 借助Mo也即移动互联网（例如随处可见的Wi-Fi）和移动用户（如各种手机、ipad 用户）之间的便捷快速的广泛传播, 优惠折扣信息更容易吸引消费者。例如人们的网络购物, 不用再每次都通过打开电脑和网页进行, 移动端购物也经常能得到更多优惠。另外, 顾客和 商家都可以从SoLoMo的一体化移动支付的过程中感受到很多便利。消费者对于SoLoMo的移 动支付已不陌生, 出门甚至只带一部手机就可以, 使用微信钱包、支付宝或余额宝、电信翼 支付、快钱等移动支付方式可以快捷买单或发起收款, 比起银行转账、取现金等流程, 不用 再漫长排队, 节省了支付时间, 提高了支付效率, 并且随着支付技术的发展, 移动支付的安 全性也得到了一定的保障。2016年兴起的网络直播, 与电子商务、微商能结合起来; 很多医 院也都实行网上预约挂号、网上缴费、网上代煎中药等便民服务; 在线教育, 例如粉笔公考 的APP也通过移动端销售更多公务员考试视频课程; 未来还有更多的领域加入移动营销、移 动支付, 因此, 移动消费市场的潜力巨大。

\section{4. 基于SoLoMo的移动电子商务精准化营销模型构建}

现在是移动互联网的时代，而移动商务一度被视为是 “下一个技术革命” ${ }^{[6]}$ ， SoLoMo 营销则成为了营销界和各个行业尤其是互联网行业极度关注的焦点。从上文的分析可知，基 于SoLoMo的移动电子商务精准化营销是一个大趋势, 谁把握住了这种 “混搭营销” 的精髓, 谁就能运筹帷幄, 决胜千里。目前, 越来越多的公司、平台、应用都在实践着对SoLoMo概念 的运用。从Facebook到人人网这样的 “So” 已经无处不在; 以LBS 为基础的各种定位和签到 的 “Lo” ，如街旁网、人人报到、百度身边、切客、新浪 “微领地” 等也纷纷产生; 智能手 机带来的各种 “Mo” 即移动互联网应用也在逐渐被开发。符合SoLoMo理念的各种网络应用 工具都已整装待发，但是，从系统和相互作用的角度分析，尝试构建一个基于SoLoMo的移动 电子商务精准化营销模型（如图1）, 进行整合营销, 才能够更加全面地运用SoLoMo的概念, 发挥更加智能化和经济腾飞指挥棒的作用。 


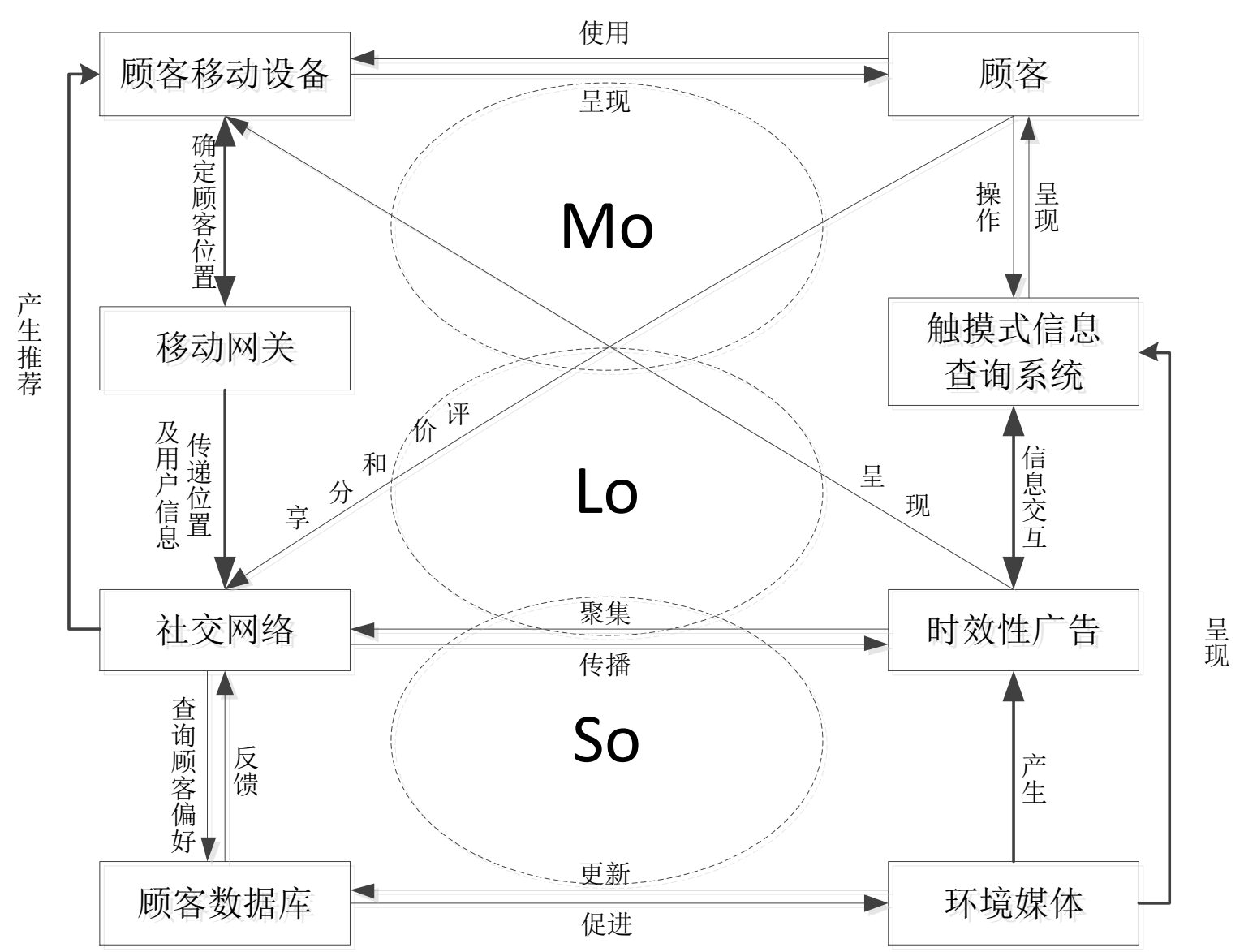

图1 基于SoLoMo的移动电子商务精准化营销模型

如上图所示, Social, Local, Mobile三者之间在移动电子商务的精准化营销模型中是相 互融合、相互作用的。顾客通过移动设备、触摸式信息查询系统等, 借助社交网络和通信功 能、互联网等与周围的一切发生信息互动, 相关的信息和踪迹被记录和整理保存在顾客数据 库中，有利于时效性广告和偏好的精准推送，同时环境媒体对这个系统产生辅助作用。

\section{1 移动化营销}

在这个过程中, Mo也即移动终端和移动网络的作用是巨大的, 其具有随客户而移动的便 捷快速特性, 移动终端上通常有很多APP, 能够提供随时随地的服务, 移动网络则使得信息 的传播速度更快, 是传统互联网所无法比拟的。现在, Wi-Fi热点的创建更加容易了, 甚至通 过手机也可以创建Wi-Fi热点。Wi-Fi也属于环境媒体中的一种。这些都保障了基于SoLoMo的 移动电子商务精准化营销的技术基础和载体。环境媒体中的二维码等图像技术或者类似于谷 歌眼镜的图像识别技术，都是要借助于移动设备的扫描来导入和呈现信息的。商家就可以利 用所呈现出的信息进行对目标受众的营销。在这方面, 微信营销就是SoLoMo营销特别是借助 Mobile营销的成功案例。商家可以发布含有商品信息或优惠信息的二维码, 通过线下活动或 传单等引导顾客用移动设备扫码关注自己的微信公众号, 便于日后的线上营销, 这也是 $\mathrm{O} 2 \mathrm{O}$ 商业模式的应用之一。再如二维码技术在博物馆的应用, 如果参观者想仔细了解展品, 就可 以通过扫码将展品的信息实时发送到移动设备 ${ }^{[7]}$, 这方面的运用还可以结合虚拟现实技术， 提供给参观者一种沉浸式的参观体验。微信的朋友圈、小视频、语音、位置共享等的实时互 动性都有利于产生更好的移动化营销效果 ${ }^{[8]}$ 。因此, 如图所示, 移动网关和移动设备是移动 营销必备的技术支撑和硬件条件。

在模型图中，顾客移动设备和移动网关的运用是移动化营销的体现。另外，环境媒体中 的虚拟现实、增强现实等技术、可穿戴设备等硬件能够使用户有身临其境的感觉，可以借助 时效性广告等推送给客户一些营销信息。这些新兴技术的呈现作为一种营销手段是极具吸引 
力的。总之, 移动设备和移动网络的广泛运用使得结合 LBS 的社交化服务成为可能, 也是快 速方便地进行社交活动的基础条件。

\subsection{LBS位置营销}

Lo也即LBS位置营销，首先，可以和电信运营商的通过GPS或者手机信号获知位置信息 合作。广告主可事先告知运营商他们的目标覆盖区域, 通过向用户发送手机短彩信推送促销 活动等, 但是这种途径要注意推送的频率和内容, 避免骚扰到用户, 触发抵抗情绪。其次, 也可以运用E-mail广告、创意网站、搜索引擎排名、CRM管理系统等从而整合电子商务系统, 加强物流、资金流、信息流的充分流动。此外，有些消费者喜欢签到服务。LBS的签到服务 让用户能在移动消费时留下消费地点痕迹。第三，根据地点的不同设置不同场景的Wi-Fi，在 用户连接上网时也可以进行对潜在的目标群体的信息推送。另外, 结合 $\mathrm{O} 2 \mathrm{O}$ 的基于位置的本 地化服务, 能够打通线上和线下, 让线上客户到线下体验, 线下的客户为线上导流, 整体上 提升用户体验。

如模型图所示，当顾客进入特定区域后，附近通讯基站的移动网关能根据顾客随身携带 的移动设备呼机、PDT等常见终端所发出的信号或者通过二维码扫描技术等确定顾客的位置 及身份（用户信息通过移动运营商的用户数据库确定），然后将其传递至社交网络上，方便 调用顾客数据库进行偏好查询和推荐。这方面可以结合大数据分析顾客的消费情况并产生更 精准的推荐。此外, 随用户地点配备一些触摸式信息查询系统等, 也能获取更多用户对地点 附近的服务的需求信息, 并将一些可能吸引顾客的信息呈现, 这对于大型商场等场合都适用。

\section{3 社会化营销}

社交网络的运用是SoLoMo营销的主要环节。社交与电子商务是相互促进的关系。通过社 交网络发展移动电子商务, 一方面可以深度挖掘数据价值找到除广告外的变现模式, 另一方 面能提供信息之外的其他价值和服务。社交圈互动性在综合平台中发展一直处于边缘地位, 社交网络或社会化媒体的运用能聚集用户为细分人群, 形成社交与电子商务相辅相成的关系。

如模型图所示，一方面，社交网络能够在顾客数据库中比对顾客信息，查询该顾客是否 有以往在商品城的购物与偏好记录, 同时调用内容库（商品介绍、时效性广告等）, 结合顾 客的地理位置和偏好选择信息, 产生TOP-N推荐, 发送至顾客移动设备上, 同时根据顾客行 为自动补充顾客数据库系统。这方面可以结合云存储技术包容更多的顾客消费数据和营销知 识、企业管理知识等, 方便随时调用。当然, 这个社交网络不仅包括现在的社会化媒体等网 络上的社交活动, 也包括传统的社交, 如面对面交流、电话或邮件沟通等。移动电子商务可 以运用顾客数据库系统实施精准营销, 也可以借鉴现实社区的群体交流进行广告投放（例如 分众传媒公司在居民的电梯内外安置广告屏幕, 展示新广告）。另一方面, 客户可以在社交 网络或虚拟社区上分享自己参与体验之后的感受或者评价, 这种口碑相传的方式也是营销的 重点。除了在网络上评价对熟人或陌生人发挥的参考作用, 在传统的社交中, 如果客户向朋 友推荐其消费过的产品或服务, 也能达到口碑营销的效果。另外, 客户也可以亲自在触摸式 信息查询系统上完成信息搜索或分享。这就涉及到环境媒体中的互动标识系统 (如 “广告牌” 技术）和表面技术了。通过社交网络或者环境媒体产生的时效性广告, 能够通过顾客移动设 备或者触摸屏推送给客户。

\section{5. 结束语}

移动电子商务正在快速发展, 结合了Social, Local和Mobile的移动电子商务营销更能实 现精准化营销, 未来的商业机会更多。移动电子商务营销者有了解移动科技含义和社交重要 程度的必要性, 理解SoLoMo是实现人与人连接、人与环境互动的移动性及信息获取的移动性 的优良途径, 这也是现代化企业实现营销管理等竞争战略优势的关键。未来, 将会有更多结 合SoLoMo的技术或应用出现，加入基于SoLoMo的移动电子商务精准化营销模型中，使其概 
念更完善, 运用更广泛, 又或者将在SoLoMo营销的三方面基础上加入其他的大方向, 那将能 更好地提高顾客体验, 满足客户的消费需求, 促进移动网络经济的发展。

\section{致谢}

本文为国家社科基金项目《虚拟社区知识组织研究》(12BTQ041)，广州市软科学研究专 项《广州市促进信息消费政策研究》(201510020008)的阶段性成果之一。

\section{References}

[1] Lu Qingping, Mobile e-commerce precision marketing model and strategy based on LBS, E-Business Journal, vol.4, pp. 20-21, 2014.

[2] Huang Chenwei, Design of location-based mobile marketing system for China commodity city, Science Technology and Engineering, vol.9, pp. 713-716, 2009.

[3] He Xi, SoLoMo: The road of cubialization marketing, China Marketing, vol.12, pp. 100-102, 2011.

[4] Cai Jianmei and Zhou Haichen, The application research on SoLoMo concept marketing based on the development of the mobile Internet, Management and Administration, vol. 2, pp. 132-134, 2015.

[5] Zhang Cuiping and Chen Jianwei, Application of O2O mode based on SoLoMo in apparel enterprise, Shandong Textile Science \& Technology, vol. 4, pp. 36-39, 2014.

[6] S. Balasubramanian, R. A.Peterson and S. L. Jarvenpaa, Exploring the implications of M-commerce for markets and marketing, Academy of Marketing Science, vol. 30, pp. 348-361, 2002.

[7] Xu Jing, WeChat marketing: A new category of SoLoMo marketing, Youth Journalist, vol. 10, pp. 78-79, 2013.

[8] Long Yaping, WeChat marketing - based on the application of SoLoMo, Market Modernization, vol. 22, pp. 88-89, 2013. 